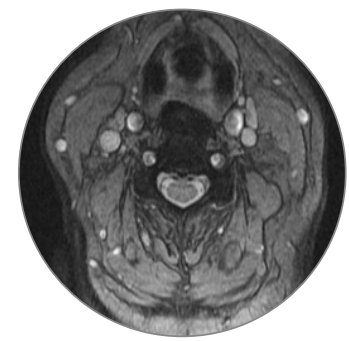

$\sum$

Palabras clave (DeCS)

Hiperostosis

Ligamentos

Vértebras cervicales

Key words (MeSH)

Hyperostosis

Ligaments

Cervical vertebrae
'Residente de cuarto año de Radiología e imágenes, Instituto Hondureño de Seguridad Social. Tegucigalpa, Honduras

2Especialista en Radiología e imágenes, Hospital Escuela Universitario. Tegucigalpa, Honduras.

${ }^{3}$ Especialista en Neurorradiología, Instituto Hondureño de Seguridad Social. Tegucigalpa, Honduras.

\section{Enfoque radiológico de la enfermedad de Forestier-Rotes-Querol. Presentación de caso}

\author{
Radiological approach to Forestier Rotes Querol disease. A case \\ report
}

\title{
Resumen
}

Se presenta el caso de una paciente femenina de 64 años de edad, con comorbilidades asociadas, quien acude al Instituto Hondureño de Seguridad Social por dolor y rigidez cervical de evolución crónica. En estudios de resonancia magnética se observa protrusión ósea con formación de puentes anteriores a los cuerpos vertebrales, que concuerda con las alteraciones halladas en tomografía y radiografía cervical. La hiperostosis esquelética difusa idiopática, o enfermedad de ForestierRotes-Querol, es una patología de la columna poco reconocida que puede cursar asintomática y desapercibida, pero ante dolor cervical la tomografía de columna es el método de estudio inicial e ideal para la valoración ósea en caso de patologías osificantes y degenerativas, que al complementar con resonancia magnética ayuda a realizar un diagnóstico preciso, para decidir entre un tratamiento quirúrgico o un manejo conservador.

\section{Summary}

The case of a 64-year-old female with associated comorbidities is presented, who attends the Honduran social security institute due to chronic cervical pain and stiffness. Magnetic resonance studies show bone protrusion with formation of anterior bridges of vertebral bodies that is consistent with the alterations visualized in tomography and cervical radiography. Idiopathic diffuse skeletal hyperostosis is a poorly recognized pathology of the spine that can be asymptomatic and unnoticed. In case of cervical pain, spinal tomography is the initial and ideal study method for bone assessment in case of ossifying and degenerative pathologies that, when complemented with magnetic resonance imaging, helps to make an accurate diagnosis and thus evaluate and decide between surgical treatment or conservative management.

\section{Introducción}

En 1950, Forestier y Rotes-Querol publicaron una serie denominada "hiperostosis anquilosante vertebral senil”. Más tarde, en 1971, Forestier y Lagier describieron la hiperostosis de la columna en adultos jóvenes omitiendo el término senil y la distinguieron como entidad nosológica separada de la espondiloartrosis y la espondilitis anquilosante (1).

La hiperostosis esquelética idiopática difusa (DISH, el inglés diffuse idiopathic skeletal hiperostosis) es una forma idiopática de artritis degenerativa que típicamente afecta a individuos mayores de 60 años de edad (con una prevalencia promedio de aproximadamente el $10 \%$ en personas con más de 50 años), de etiología desconocida. La prevalencia mundial oscila entre el 2,9\% y el $42 \%$ (2).

Esta patología es más frecuente en hombres que en mujeres, con una relación 2/1, diferencia que disminuye con la edad; se limita a la columna vertebral, pero, a la vez, puede afectar múltiples sitios periféricos de forma independiente.
Las osificaciones de entesis extraespinales son frecuentes, y cuando se encuentran aisladas pueden conducir al diagnóstico de DISH. Se ha descrito la coexistencia de esta entidad con espondilitis anquilosante(3).

Si bien la mayoría de los pacientes con afectación cervical son asintomáticos o solo tienen limitación de la movilidad o dolor inespecífico, existen distintos informes de pacientes con síntomas secundarios a la compresión del esófago y la vía aérea, como disfagia, estridor, disfonía y dificultades con la intubación o endoscopia, por lo cual el diagnóstico de esta entidad se debe sospechar como una posibilidad en los casos de disfagia, una vez descartadas otras causas de disfagia más frecuentes $(1,4)$.

Los síntomas de la enfermedad suelen estar determinados por las osificaciones del periostio, más frecuente en las zonas de inserciones óseas de los ligamentos y tendones; la localización más habitual es en la región torácica de la colunna, con escasa frecuencia, en la zona cervical (5). 
La excelente resolución espacial de la tomografía computarizada (TC) permite una evaluación precisa de las articulaciones facetarias, que es un aspecto importante para diferenciar la DISH de la espondilitis anquilosante.

El caso que aquí se presenta tiene como objetivo dar a conocer los diferentes métodos diagnósticos para esta patología de la columna vertebral y, en este caso en particular, la complementación de TC y resonancia magnética $(\mathrm{RM})$, ayudará en la discriminación de patología ósea-discal.

\section{Presentación del caso}

Paciente de sexo femenino de 64 años de edad con antecedente de diabetes, hipertensión arterial e hipotiroidismo, quien se presenta con sintomatología caracterizada por cervicalgia y rigidez de la columna cervical de evolución crónica.
Al examen físico neurológico se observa marcha con lateralización a la derecha, dolor y rigidez cervical con limitación de la movilidad. Reflejos osteotendinosos, vibración y sensibilidad conservados en los miembros superiores. Se le realizan estudios radiológicos, de TC y RM para descartar patología discocervical degenerativa.

La TC cervical con reconstrucción sagital y cortes axiales, muestra osificación a lo largo de la cara anterior de los cuerpos vertebrales desde C2 hasta C7 con preservación de los espacios intervertebrales, lo cual concuerda con lo visualizado en radiografía cervical en proyección sagital (figura 1).

La RM de la columna cervical mostró crecimiento óseo en el borde anterior de los cuerpos vertebrales que protruye al espacio prevertebral y que es posterior al esófago y laringe, correspondiente a puentes entre las vértebras, que no comprometen el espacio intervertebral y sin evidencia de patología discal o radicular (figura 2).
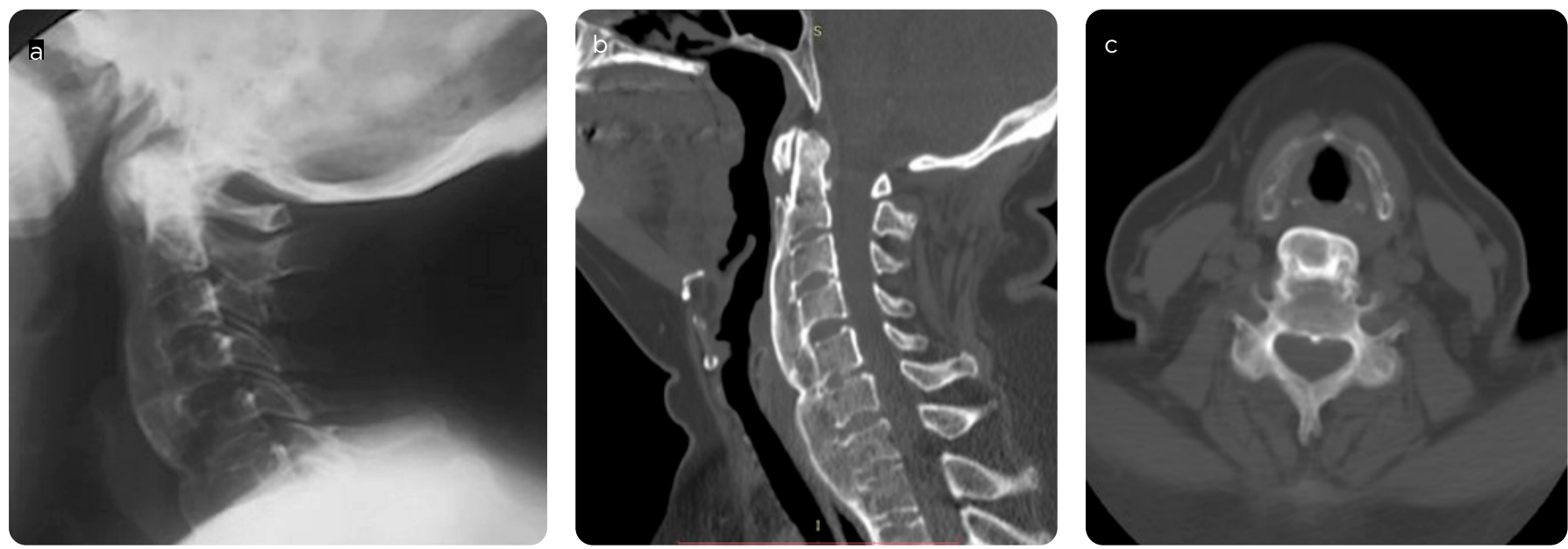

Figura 1. a) RX de la columna cervical, proyección lateral: pérdida de lordosis y extensa hiperostosis anterior al ligamento longitudinal anterior. b y c) TC de columna cervical reconstrucción sagital y corte axial: osificación a lo largo de la cara anterior de los cuerpos vertebrales desde C2 hasta C7 con preservación de los espacios intervertebrales.
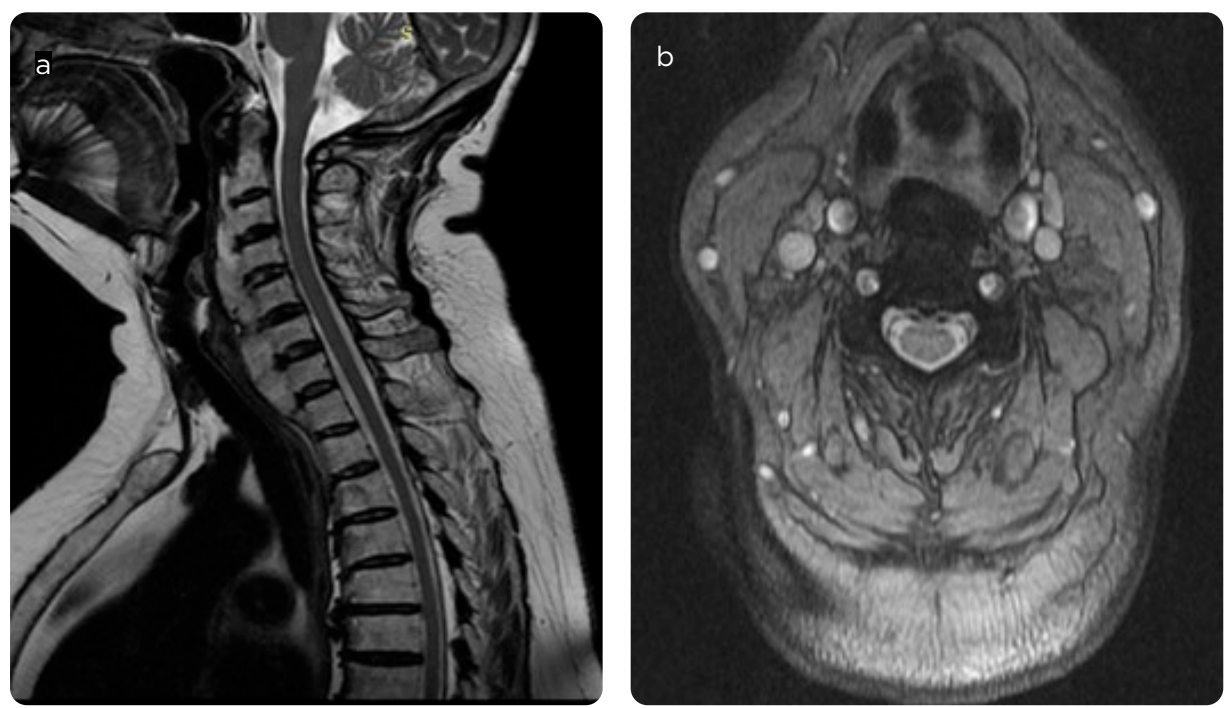

Figura 2. a y b) RM Secuencia con información T2 sagital y secuencia MERGE (multiple echo for recombined gradient echo) axial. Crecimiento óseo en borde anterior de cuerpos vertebrales que protruye al espacio prevertebral anterior y que es posterior al esófago y laringe. Corresponde a puentes entre las vértebras que no comprometen el espacio intervertebral, sin evidencia de patología discal , compresión medular ni radicular. 


\section{Discusión}

La hiperostosis esquelética idiopática difusa (DISH) es un trastorno caracterizado por osificación y calcificación a lo largo de la cara anterolateral de los cuerpos vertebrales y entesis periféricas (6).

Se han sugerido varios agentes causales, como diabetes mellitus, obesidad, vejez, hiperuricemia, hipertensión arterial, hiperinsulinemia y el factor de crecimiento similar a la insulina. Algunos trabajos también han citado traumas, ocupación, fluorosis, enfermedades infecciosas, origen autoinmune (HLA-B27, HLA-B5, HLA-A11), neuroartropatías e hipervitaminosis A como causas primarias $(2,7)$.

En 1976, Resnick y Niwayama (8) propusieron los criterios de diagnóstico para DISH en la columna basados en características radiológicas: 1) osificación de al menos cuatro cuerpos vertebrales contiguos; 2) preservación relativa del espacio del disco intervertebral, y 3) ausencia de anquilosis ósea de articulaciones apofisarias y de erosión articular, esclerosis o fusión ósea intraarticular sacroilíaca

Los dos primeros criterios ayudan a distinguirla de la espondilosis y el tercero, de la espondilitis anquilosante. Aunque en estudios recientes en pacientes con DISH intervenidos de la columna lumbar, la formación de puente óseo anterior y anquilosis de la articulación sacroilíaca eran más frecuentes.

En ocasiones se dificulta el diagnóstico diferencial, porque pueden existir ambas patologías. También se pueden observar hiperostosis en el tobillo, en la cara posterior del calcáneo y en el codo, en la inserción del tríceps en el olécranon $(2,6)$.

La TC de columna vertebral muestra la osificación a lo largo de la cara anterior de los cuerpos vertebrales, y las reconstrucciones en planos coronal y sagital suelen mostrar el patrón clásico de osificación y puentes óseos. La RM por lo general, no está indicada inicialmente en la DISH. Sin embargo, puede ser valiosa cuando la osificación del ligamento longitudinal posterior causa síntomas neurológicos, pues permite determinar la extensión de dicha osificación, el efecto de la masa sobre el saco tecal y la compresión del cordón medular (2).

En el caso aquí estudiado, el paciente tenía algunos factores de riesgo y la hiperostosis se localizó en la región cervical, el lugar de menor ocurrencia; pero hay casos en la literatura de mayor complejidad, como el descrito por Ito et al., donde el paciente presentó hiperostosis del ligamento longitudinal anterior y posterior con estenosis del canal medular, además, hiperostosis de los ligamentos entre las apófisis espinosas y osteofitosis anterior que dificultaban la deglución aun después de la primera intervención quirúrgica (7).

El diagnóstico diferencial incluye espondilosis deformante, la espondilitis anquilosante, la artritis psoriásica; además, la fluorosis, la osteomalacia, la acromegalia, la paquidermoperiostitis, la hipofosfatemia y el hipoparatiroidismo. El manejo inicial del DISH debe ser conservador; consiste en cambios en la dieta, antiinflamatorios, relajantes musculares, corticoesteroides y cambios posturales durante el proceso de deglución. La cirugía, para los casos progresivos o muy sintomáticos, suele realizarse por vía anterior con resultados favorables, pero tiene el inconveniente de que la enfermedad puede recurrir, sobre todo cuando se ejecuta en los segmentos de mayor movilidad(8-10). La enfermedad en general, tiene buen pronóstico; se requiere cirugía en aquellos casos con afección cervical que involucra las vías digestivas o respiratorias altas.

\section{Conclusión}

La enfermedad de Forestier-Rotes-Querol, una causa inusual de dolor cervical, puede pasar desapercibida y ser un hallazgo incidental en estudios de imágenes; sin embargo, el abordaje radiológico es ideal para descartar causas degenerativas, entre otras, y es una herramienta para su diagnóstico rápido y eficaz. Entre las modalidades diagnósticas, la TC como método inicial ayuda a evaluar el compromiso óseo, para considerar tratamiento conservador o quirúrgico. La RM es complementaria para evaluar afectación de otras estructuras y darle un panorama amplio al médico tratante.

\section{Referencias}

1. Aguilar-Araiza MA, De la Torre-González DM, Ortiz-Rojas F. Hiperostosis esquelética idiopática difusa. Rev Hosp Jua Mex. 2013;80(2):141-4.

2. Ghisi J, Plata C. Resolución del caso. Rev. Asoc Argent Ortop Traumatol. 2018;83(2): 131-3. Doi: http://dx.doi.org/10.15417/issn.1852-7434.2018.83.2.838

3. Orden AO. La hiperostosis esquelética idiopática difusa (DISH) como expresión de un proceso sistémico. Rev Arg Reumatol. 2017;28(2):29-33.

4. Urrutia J, Bernardín A, Morales C, Millán R. Disfagia cervical espondilótica por hiperostosis esquelética difusa idiopática en un paciente joven. Rev Med Chile. 2013;141:803-6.

5. Sánchez González F, Benito Arroyo I, Urbano Urbano J, Paulino Herrera A. Enfermedad de Forestier-Rotes Querol (hiperostosis difusa esquelética idiopática) SEMERGEN. 2006;32(9):461-3.

6. Yahara Y, Yasuda T, Kawaguchi Y. et al. Sacroiliac joint variation associated with diffuse idiopathic skeletal hyperostosis. BMC Musculoskeletal Disorders. 2020;21:93. Doi: https://doi.org/10.1186/s12891-020-3105-z

7. Ito K, Kadono I, Okada T, Hishida A, Ando K, et al. Dysphagia after C2-7 in situ posterior fusion in a patient with diffuse idiopathic skeletal hyperostosis: Case report of a rare presentation. Spine Surg Relat Res. 2019;3(3):270-3. doi: 10.22603/ssrr.2018-0083

8. Resnick D, Niwayama G. Radiographic and pathologic features of spinal involvement in DISH. Radiology. 1976;119:559-68. doi: http://dx.doi.org/10.1148/119.3.559.

9. Valdez C, Valdez A. Enfermedad de Forestier en paciente mayor de 65 años: reporte de caso con seguimiento a 6 años de manejo conservador. Rev Med Chile. 2018;146: 1493-6.

10. Domínguez-Gasca LG, Álvarez-Rodríguez M, Domínguez-Carrillo LG. Hiperostosis esquelética idiopática difusa. Enfermedad de Forestier. Rev Med Clínica Mayo. 2019;3(2):115-8.

\section{Correspondencia}

Sandra María Cárcamo Mejía

Residencial Prados universitarios

Tegucigalpa, Honduras

sandriscarcamo87@gmail.com

Recibido para evaluación: 20 de enero de 2021

Aceptado para publicación: 4 de mayo de 2021 